\title{
Ti-Ni SMA thin film microactuators made on a Si substrate
}

\author{
S. Miyazaki, V.H. No, M. Taniguchi and H. Suzuki \\ Institute of Materials Science, University of Tsukuba, Tsukuba, Ibaraki 305-8573, Japan
}

\begin{abstract}
:
Among several types of performance materials proposed for fabricating microactuators, Ti-Ni shape memory alloys have great advantages such as large deformation and recovery force. The intrinsic disadvantage of shape memory alloys, the slow response due to the limitation of cooling rate can be greatly improved in micro size actuators, because the surface/volume ratio of materials will drastically increase in thin films so that cooling becomes efficient. Therefore, the development of $\mathrm{Ti}-\mathrm{Ni}$ shape memory thin films has been proposed in the field of micromachines.

In the present investigation, $\mathrm{Ti}-\mathrm{Ni}$ shape memory alloy thin films were deposited by sputtering on $\mathrm{Si}$ substrates covered by $\mathrm{SiO} 2$ layer. The $\mathrm{Ti}-\mathrm{Ni}$ thin films were crystallized by heat-treatment in order to achieve high transformation temperatures. The Ms and As temperatures were $340 \mathrm{~K}$ and $365 \mathrm{~K}$,respectively. This $\mathrm{Ms}$ is the highest temperature achievable in the binary Ti-Ni alloy and about $50 \mathrm{~K}$ higher than room temperature, enabling that a high frequency response is achieved. Square diaphragm type microactuators were fabricated by the photolithography method with anisotropic etching applied to the $\mathrm{Si}$ substrate. The dimension of the sides of the squares ranges between 100 and 1,000 micronmeters. The structure of the diaphragm consists of a 1.5 micronmeters thick Ti-Ni film and a 1.0 micronmeter thick SiO2 layer. Joule heating and natural cooling methods were applied to move the actuators. The microactuators moved up and down upon cooling and heating, respectively. The highest position at the center of the actuator was about 30 micronmeters, while the lowest was 0 micronmeters. The highest frequency detected was $100 \mathrm{~Hz}$.
\end{abstract}

